Exploring students' understanding of reference frames and time in Galilean and special relativity

This article has been downloaded from IOPscience. Please scroll down to see the full text article.

2010 Eur. J. Phys. 311527

(http://iopscience.iop.org/0143-0807/31/6/017)

View the table of contents for this issue, or go to the journal homepage for more

Download details:

IP Address: 78.251.213.60

The article was downloaded on 21/10/2010 at 07:48

Please note that terms and conditions apply. 


\title{
Exploring students' understanding of reference frames and time in Galilean and special relativity
}

\author{
C de Hosson ${ }^{1}$, I Kermen $^{2}$ and E Parizot ${ }^{3}$ \\ ${ }^{1}$ Laboratoire de didactique André Revuz, Physics Department, Université Paris Diderot, \\ France \\ ${ }^{2}$ Laboratoire de didactique André Revuz, Université d'Artois, France \\ ${ }^{3}$ Laboratoire Astroparticule et cosmologie, Physics Department, Université Paris Diderot, \\ France \\ E-mail: cecile.dehosson@univ-paris-diderot.fr
}

Received 5 July 2010, in final form 25 August 2010

Published 20 October 2010

Online at stacks.iop.org/EJP/31/1527

\begin{abstract}
This paper aims at exploring prospective physics teachers' reasoning associated with the concepts of reference frame, time and event which form the framework of the classical kinematics and that of the relativistic kinematics. About 100 prospective physics teachers were surveyed by means of a questionnaire involving classical kinematics situations and relativistic ones. The analysis of the answers shows a deep lack of understanding of both concepts of reference frame and event. Some students think that events may be simultaneous for an observer and not simultaneous for another one, even when both observers are located in the same reference frame. Most of the students surveyed cannot give an answer only depending on the location of the observer when his/her velocity is mentioned as if the movement contaminated the event. This lack of understanding is embodied in reasoning implemented by the population surveyed to address classical kinematics questions and seems to form a major obstacle to grasping relativistic kinematics.
\end{abstract}

\section{Introduction}

This research takes place within the context of the EVEILS research project (French acronym for Virtual Spaces for the Education and Illustration of Science). This project aims at exploring the innovating potential of virtual reality (VR) in several areas of science through an interdisciplinary approach involving physicists, VR specialists and physics education researchers. The project exploits advanced interfaces in order to 'immerse' a student in unusual phenomena otherwise inaccessible to human experience. The exploration of the 
cognitive modifications and pedagogical advantages associated with the 'immersion' is part of the main goals of EVEILS. This educational aspect makes EVEILS quite specific among the research programs devoted to computer simulations associated with VR (Savage et al 2007).

The relativistic structure of spacetime cannot be grasped through direct, sensible human experience (Einstein and Infeld 1938). Thus, we decided to apply VR to special relativity by favouring the understanding of perceptible relativistic effects and the construction of relativistic concepts thanks to appropriate learning scenarios. In order to do so, 'didactic engineering' as a framework for the development of teaching materials is adopted (Artigue 1994). This implies a prior analysis of the cognitive constraints and difficulties related to the learning of a given concept. In that perspective, we investigated the students' understanding of relativistic situations. Our study is part of a research context still largely unexplored.

\section{Prior studies}

What do we learn from previous studies about understanding special relativity? These studies are not very numerous but they showed that students fail in defining and using the concept of event (Hewson 1982) and thus confuse the instant of an event and the instant of the reception of that event by an observer (Scherr et al 2001, Scherr 2007). Moreover, they use 'spontaneous' kinematics lines of reasoning (such as absolute motion, distances and velocities) to explain mechanical phenomena in both classical and special relativity frameworks (Saltiel and Malgrange 1980, Villani and Pacca 1987). These results were confirmed by a recent study conducted with 10th grade students in Greece (Dimitriadi et al 2009). Students think that simultaneity is absolute and independent of relative motions (Villani and Pacca 1987, Scherr et al 2001). Students fail in understanding the concept of reference frame: each observer constitutes a distinct reference frame, and if observers are in the same location, they belong to the same reference frame (Scherr et al 2001).

\section{Focus of the research}

The transition from classical to relativistic kinematics requires a radical change in the conceptual framework. In the theory of special relativity, $c$ is a constant that connects space and time in the unified structure of spacetime. The speed of light is equal to that constant and thus is invariant with respect to any inertial reference frame. Besides, the simultaneity of two events is not absolute (two events at different locations that occur at the same time in a given reference frame are not simultaneous in all other reference frames). Assuming this change in the conceptual framework requires a sound knowledge of the concepts of reference frame and event that underpin the laws of classical kinematics. A reference frame can be defined as a set of observers at rest relative to each other. These observers determine the same distances and time delays between any set of events where an event is defined as a fact that occurs at a given location in space and at a given instant in time.

A poor understanding of the concepts of reference frame and event can be a major obstacle to moving from the classical to the relativistic conceptual framework. The general purpose of our investigation is to analyse how prospective physics teachers in physics and chemistry understand and use the notions of reference frame and event. This research aims at identifying the types of reasoning implemented by prospective physics teachers faced with situations of classical and relativistic kinematics. 


\section{Overview of the research}

\subsection{Student populations}

The research was conducted in France from May 2009 to January 2010. The study has involved 94 prospective physics and chemistry teachers (in France, physics teachers in lower and upper secondary school have to graduate both in physics and chemistry and teach both subjects) from five different teacher training institutes (IUFM). All are third-year graduate students in chemistry, or in physics or in physics and chemistry. The population includes at least 44 students who studied special relativity in their physics courses.

\subsection{Research method}

The research was conducted through the analysis of the students' responses to eight multiple choice questions including a request for justification. The questionnaire ('the situation of the bridge') is presented in appendix A (the ${ }^{* *}$ ' symbol shows the expected answers). The questions are not all involving special relativity. Only two of them (e.g. Q6 and Q8) require the implementation of relativistic reasoning. The other questions resort to Galilean kinematics. It is worth noting that throughout the questionnaire, the understanding of the concept of event is put to the test through the situations where either only the position of the observers is involved or only their velocity. Questions the answer to which depends only on the position of the observers are about Galilean kinematics, and questions the answer to which depends only on the velocity of the observers are related to special relativity. Moreover, for each observer, two different questions are asked: one requiring an answer about the reception of signals, the other requiring an answer about the emission of signals.

\subsection{Presentation of the research questions}

Our analysis rests on two dimensions: a deductive one and an inductive one. The 'deductive' dimension stems from the content analysis and the analysis of the students' difficulties. By 'inductive' dimension we mean that some categories of justification are not defined a priori but are defined after examining data, they are emergent categories of justification (Strauss and Corbin 1990). The a priori analysis involving four research questions is now presented.

4.3.1. Not specifically relativistic questions. Through the 'Galilean' questions, we seek to identify the difficulties associated with the concept of reference frame and the possible existence of confusion between the instant at which an event occurs and the instant at which this event is perceived by an observer (Scherr et al 2001). Our first research questions (RQn) are as follows:

- RQ1: How do the students understand the concept of reference frame?

- RQ1a: Is a reference frame understood as a set of observers at rest relative to each other?

- RQ1b: Do the students identify the questions requiring an answer depending on the location and not on the velocity of the observer, and vice versa?

- RQ2: Is the instant at which an event occurs in a given reference frame confused with that at which it is perceived by an observer? 
The answer to RQ1a is provided by checking the consistency of answers to questions Q2 and Q4 and to questions Q6 and Q8. Indeed, Alice (A), Bernard (B), Cecile (C) and Denis (D) belong to the same reference frame, the reference frame of the bridge $\mathrm{Rb}$ (they are at rest relative to each other). Therefore, for these four observers, each event ('A triggers the flash' and 'B triggers the flash') occurs at the same value of their common time coordinate and of their common space coordinates. Similarly, Etienne $(E)$ and Fanny $(F)$ belong to the same reference frame, the reference frame of the scooter Rs (different from Rb). Thus, the statements about the spacetime coordinates of events should be identical for $E$ and $F$. Therefore we expect the students to produce identical responses to questions Q2 and Q4 on the one hand, and to questions Q6 and Q8 on the other hand. Moreover, if the students agree with the idea that two signals are received in the same order by two observers at the same coordinates of space and time, they will provide identical answers to Q1 and Q5 on the one hand, and to Q3 and Q7 on the other hand. This will provide elements to answer RQ1b.

To answer RQ2 we ask the students whether two events 'A triggers the flash' and 'B triggers the flash' are perceived at the same time by two different observers belonging to the same reference frame. The time at which an event occurs is distinct from the instant at which the event is perceived by any observer. If the instant at which an event occurs in a given reference frame is confused with that at which it is perceived by an observer, then one may expect justifications such as: ' $\mathrm{C}$ receives the flash emitted by $\mathrm{A}$ at the same time as the one emitted by B, thus the two flashes were emitted at the same time' in $\mathrm{Q} 2$ and such as ' $\mathrm{D}$ receives the flash emitted by A before that emitted by B, so the event $A$ triggers the flash happens before the event $B$ triggers the flash' in Q4. Justifications of the same type may be provided in Q6 and Q8.

4.3.2. Specifically relativistic questions $(Q 6, Q 8)$. Through these questions, we seek to analyse the students' ability to identify the need to change the interpretive framework and to determine the extent to which the students use the classical kinematics framework. These questions bring into play situations where the invariance of the speed of light and the relativity of simultaneity ought to be implemented. We add another research question to the aforementioned ones:

- RQ3: How does classical kinematics reasoning become included in the resolving of relativistic questions?

In response to RQ3 we try to see whether the students use the classical kinematics framework to explain relativistic situations. We analyse how the students answer Q6 and Q8. The application of the classical kinematics framework will certainly imply that the students reply that the two events 'A triggers the flash' and 'B triggers the flash' are simultaneous in Rb and Rs. Doing so, they will probably tick box a) in Q6 and Q8. This analysis should provide an indication of the way that the students understand the concept of reference frame. Indeed, if the students consider that two pairs of observers ( $C$ and $E$ on the one hand, $D$ and $F$ on the other hand) being at the same coordinates of space and time agree on the simultaneity of two events (A triggers the flash and B triggers the flash), we could probably interpret that as 'two observers being at the same coordinates of space and time belong to the same reference frame', or as 'being in two different reference frames does not imply any difference in the time interval between two events'. 


\section{Results and discussion}

In this section we present analyses in order to provide elements to answer the research question. The distribution of answers and choices to all questions is set out in appendix B.

Firstly we question the students' understanding of a reference frame. In particular we try to see whether the students consider that different observers at rest relative to each other pertain to the same reference frame. Therefore we examine whether the answers to Q2 and Q4 on the one hand and to Q6 and Q8 on the other hand are consistent. If the students have a sound understanding of the concept of reference frame, they should make consistent choices for each set of two questions and give relevant justifications. For Q2 and Q4, the choice is 'the flashes have been emitted at the same time' (box a)) for both questions and a relevant justification to Q4 mentions that C and D belong to the same reference frame. $15 \%$ of the students did so. For Q6 and Q8, given the dispersal of justifications we choose to focus on the expected justification before taking into account the correctness of the choice. So, only $4 \%$ of the students justify the choice they make to answer Q8 (whatever it is) by correctly referring to the explanation they provide to Q6 or by starting that $E$ and $F$ belong to the same reference frame. $2 \%$ give such a justification and make the correct choice, box c), the others make a wrong choice. Summarising this analysis, $4 \%$ of the students give a justification resorting to observers pertaining to the same reference frame for the two sets of questions. And only $2 \%$ of them make a correct choice for the two sets of questions. In the light of this result it appears that considering a reference frame as a set of observers at rest relative to each other is not an operative concept for the population surveyed.

Another issue related to the understanding of the concept of reference frame concerns the reception of signals by two different observers located at the same point of space and time at a given point of their timeline, but not at rest relative to each other. Therefore, we put into perspective the consistency of the answers to Q1 and Q5 (the two observers are Cecile and Etienne) on the one hand and to Q3 and Q7 (the two observers are Denis and Fanny) on the other hand. 28\% of the students provide correct justifications to Q5 (same place or same place and instant) and 65\% to Q1. Among them 23\% (22 students) give a correct response and justification to Q1 and resort to the similarity of the positions of Cecile and Etienne to justify the correct choice to Q5. Regarding the other set of two questions, it is worth noting that all the students who correctly answer Q7 also give a right response to Q3. Nevertheless one notes the decreasing of the percentage of the expected answer; 54\% give a correct justification (and right choice) to Q3 whereas only 23\% respond well to Q7. This is somewhat surprising because a correct justification to both questions resorts to the same notion since the two observers, Denis and Fanny, are at the same point of space and time when the flash from Alice is received, which is for both of them the first signal to be received. We may tentatively interpret the decreasing of the percentage of the right line of reasoning as having something to do with the velocity of Fanny. As mentioned above, the students who correctly answer Q3 and do not correctly answer Q7 seem to take into account Fanny's velocity, although it is not a relevant argument, as if the movement 'contaminated' the event. Now if we put together both parts of the ongoing analysis, we determine that 13\% (12 students) of the students answer Q1 and Q5 in a consistent way on the one hand and Q3 and Q7 on the other hand. It appears that most of the students surveyed cannot give an answer only depending on the location of the observer when his/her velocity is mentioned, which reveals a difficulty associated with the notion of event and its independence of the reference frame.

Our second research question focuses on the confusion that students make between the instant at which an event occurs in a given reference frame and the instant at which it is perceived by an observer. After analysing all the justifications, we can say that some students 
write that the instant at which a signal is emitted depends on the instant at which it is received by the observer. In other words, these students think that because they know the instant of the reception of signals by an observer then it is possible to say at which instant the signals were emitted. This tendency could be interpreted as a manifestation of confusion between the instant of emission and the instant of reception of light.

Previous analyses show that $14 \%$ of the students tick the expected answer for Q2 (both flashes have been emitted at the same time) and base their reasoning on their justification to Q1 and provide a justification that makes the emission depend on the reception and that $18 \%$ of the students respond incorrectly to Q4 that Alice's flash has been emitted first and justify this by evoking the moment of its reception or the fact that the distance from Denis to Alice is smaller than the distance to Bernard. Five students belong to both populations; it means that $26 \%$ ( 24 students) of the students write this type of justification at least once. For these students the instant at which the flashes are emitted is explicitly linked to the instant at which they are perceived by D without explicitly mentioning the covered distances. Similarly, approximately one-quarter of the students surveyed who justify their choices to questions Q6 and Q8 do so by conditioning the instant of the emission of both flashes to the instant at which the flashes are perceived by Etienne (Q6) and Fanny (Q8). As the students do not mention the distances covered by the photons emitted by both flashes, we are not able to properly answer this research question.

In order to answer RQ3 we wonder whether the students use the classical kinematics framework to explain relativistic situations. We attempt to determine to what extent the simultaneity of events is seen as absolute (answers to Q6 and Q8). 29\% of the students surveyed answer the flashes were emitted at the same time in Etienne's reference frame (Q6) and 27\% in Fanny's reference frame (Q8). If we cross-reference these responses we find that nineteen students (20\% of the students surveyed) answer both questions this way (box a)). Seven students among them provide a justification using the classical kinematics framework. As an example, student number 77 answers 'the moment of emission does not depend on the reference frame' in Q6 and 'the initial moment is always the same' in Q8. Seven others (among the nineteen quoted above) do not justify their choice. This means that fourteen students (15\% of the students surveyed) give the same type of answer to both Q6 and Q8 (answer justified within the classical kinematics framework or not justified) and among them nine students had attended a course on special relativity. We can say that among the students who answer these two questions (whatever their choice of response), one third (33\%) use classical kinematics reasoning (with or without justification). This percentage is far higher than the one of correct answers (12\%) of which only two are properly justified in Q6 and Q8. We can note that among the students (11) who choose the correct answer, six had attended a course on special relativity, and two of them give a correct justification. This perspective suggests that the framework of classical kinematics remains dominant even after special relativity instruction.

Now we discuss the issue of non-simultaneity. The fact that $10 \%$ of the students answer that Alice's flash has been emitted first for both Q6 and Q8 (box b) ticked) or 13\% respond that Bernard's flash has been emitted first for both Q6 and Q8 (box c) ticked, which is the expected choice) could be an indication that simultaneity is not considered by $23 \%$ of the students surveyed as absolute. Nevertheless, we wonder to what extent their choices have been governed by the confusion they could make between the instant when a signal is emitted and the instant it is perceived by an observer. Indeed, if the students associate the order in which both signals are emitted with that in which both signals are received by the observers, then the non-simultaneity of the signal emissions does not appear as a consequence of a change of kinematic framework and is therefore not a problem. In other words, for these students, the non-simultaneity is not questionable since it is associated with a 
given order of signal perception and not as a consequence of the relativistic framework. This can be explored through the consistency of answers to both Q5 and Q6 on the one hand and to both Q7 and Q8 on the other hand. In fact, the low number of justifications in Q6 and Q.2.8 is an obstacle to a relevant exploration. Nevertheless, it can be noticed that five students who consider the flashes have been emitted at different instants (tick box c) (or b) for both Q6 and Q8) also respond the flashes have been perceived at different instants by Fanny and Etienne (tick box c) (or b) for both Q5 and Q7) and justify their choice associating the instant at which both signals are emitted with that at which they are perceived by the observers. As an example, student 62 answers Q5 saying 'Etienne turns his back on Alice, when he comes abreast of Cecile, the light is reaching him; thus, it is at this moment he will see her flash, whereas he saw Bernard's flash right at the beginning', and Q6 saying 'Etienne sees the flash emitted by Bernard first so it has been emitted first'. This student maintains his reasoning for Q7 and Q8. To sum up, $19 \%$ of the students surveyed do not consider that the two signals have been emitted simultaneously for both Q6 and Q8. 6\% among them condition their choice to the justification provided in Q5 or Q7 considering that the two signals have not been perceived by Etienne (or Fanny) at the same time. 3\% provide a correct justification and choose the expected box, and $14 \%$ of the students ticking box b) or c) in both Q6 and Q8 do not provide any justification or a justification that is difficult to interpret. For these students, we may assume that they have no problem with the fact that the simultaneity of two events is not absolute, when one moves from one reference frame to another, because they do not perceive simultaneity as universal within a single reference frame. The simultaneity of two events thus appears as relative as the simultaneity of their perception.

\section{Conclusion}

A mathematical tool such as 'the Lorentz transformation' is never written correctly if it is mentioned and other tools such as spacetime diagrams are not used; thus, we can say that mathematical tools are not operative when they are known (Scherr et al 2001).

The population surveyed is composed of prospective physics teachers, about half of whom have not attended a course on special relativity. Moreover the prospective physics teachers who had attended a course on special relativity had done so two or three years previously. In contrast, the other studies we refer to (Dimitriadi et al 2009, Scherr et al 2001, Villani and Pacca 1987) involved students surveyed a short time after teaching, who used relativistic arguments such as time dilation and length contraction. The elapsed time between the special relativity course and our questionnaire is quite long. Furthermore, during their training, prospective physics teachers do not have to take special relativity concepts into account. These elements may explain why so few students use relativistic arguments in our study.

Another aspect is rather surprising, as we advance in the questionnaire the number of non-responses increases (Q5: 15\%; Q6: 24\%; Q7: 32\%; Q8: 44\%). Is it due to a weariness effect or does it reflect the perplexity of some students? We believe that a positive response to both questions may be a good interpretation of this trend.

The cross analysis of Q2 and Q4 on the one hand and of Q6 and Q8 on the other hand shows that considering a reference frame as a set of observers at rest relative to each other is not an operative concept for the population surveyed. This result echoes Scherr's et al (2001). The search for consistency between answers to Q3 and Q7 on the one hand and between answers to Q1 and Q5 on the other hand shows that most of the students surveyed cannot give an answer depending only on the location of the observer when his/her velocity is mentioned as if the movement contaminated the event. 
Some students confuse the instant at which signals are perceived and the instant at which signals are emitted. This tendency is not so pronounced maybe because our questionnaire explicitly differentiates both phenomena (emission from perception). However, the dependence between the instant of emission and the instant of perception seems to erase the difficulty associated with the relativity of simultaneity. Indeed, some students think that events may be simultaneous for an observer and not simultaneous for another one, even when both observers are located in the same reference frame. This is due to the fact that they associate the order in which two events are perceived and the order in which these events occur. The order of perception conditions the order of emission when it should be the opposite (with explicit consideration of the distance between the events and the observer), as if causality would apply from future to past. Thus, if one does not distinguish emission from perception then the issue of the non-simultaneity is not a problem but the concepts involved in special relativity are totally ignored.

The classical kinematics framework is still dominant to resolve relativistic situations and students who have attended special relativity course are not capable of producing a significantly better answer than those who have not. Finally, we highlighted deep misunderstandings of the comprehension of the spacetime structure of classical kinematics which constitute later on a major obstacle for the grasping of relativistic concepts.

\section{Acknowledgment}

EVEILS is a research project supported by the French National Research Agency (ANR) and conducted under the responsibility of Professor Etienne Parizot.

\section{Appendix A. Test}

Q1. It is night. Two tourists Alice and Bernard stand motionless facing each other at each end of the same bridge. Their daughter Cecile stands in the middle of the bridge. At a given moment, she makes them sign to take a picture with flash (considering that the reaction times of Alice and Bernard are identical). Does Cecile see the light flashes at the same time?

(a) Yes, she sees the light flashes at the same time*

(b) No, she sees the flash of Alice first

(c) No, she sees the flash of Bernard first

(d) I do not know

Justify

Q2. Have the light flashes of Alice and Bernard been emitted at the same time in the reference frame of Cecile?

(a) Yes, both flashes have been emitted at the same time*

(b) No, the flash of Alice has been emitted first

(c) No, the flash of Bernard has been emitted first

(d) I do not know

Justify 
Q3. Denis is standing motionless on the bridge between Alice and Cecile. Does Denis perceive the light flashes at the same time?

(a) Yes, he perceives the light flashes at the same time

(b) No, he perceives the flash of Alice first*

(c) No, he perceives the flash of Bernard first

(d) I do not know

Justify

Q4. Have the light flashes of Alice and Bernard been emitted at the same time in the reference frame of Denis?

(a) Yes, both flashes have been emitted at the same time*

(b) No, the flash of Alice has been emitted first

(c) No, the flash of Bernard has been emitted first

(d) I do not know

Justify

Q5. Etienne is crossing the bridge on a relativistic scooter at a constant velocity $(v=0.8 c)$ relative to the ground. He is going from Alice to Bernard and reaches abreast of Cecile at the very moment she receives the light emitted by the two flashes. Does Etienne perceive the light flashes at the same time?

(a) Yes, he perceives the light flashes at the same time*

(b) No, he perceives the flash of Alice first

(c) No, he perceives the flash of Bernard first

(d) I do not know

Justify

Q6. Have the light flashes of Alice and Bernard been emitted at the same time in the reference frame of Etienne?

(a) Yes, both flashes have been emitted at the same time

(b) No, the flash of Alice has been emitted first

(c) No, the flash of Bernard has been emitted first*

(d) I do not know

Justify

Q7. Fanny is crossing the bridge on a second relativistic scooter at the same velocity and in the same direction as Etienne's. She reaches abreast of Denis at the very moment he receives the light emitted by Alice. Does Fanny perceive the light flashes at the same time?

(a) Yes, she perceives the light flashes at the same time

(b) No, she perceives the flash of Alice first*

(c) No, she perceives the flash of Bernard first

(d) I do not know

Justify

Q8. Have both light flashes been emitted at the same time in the reference frame of Fanny?

(a) Yes, both flashes have been emitted at the same time

(b) No, the flash of Alice has been emitted first

(c) No, the flash of Bernard has been emitted first*

(d) I do not know

Justify 
Appendix B. Distribution of answers and choices

Table B1. Distribution of answers to Q1, Q2, Q3 and Q4.

\begin{tabular}{|c|c|c|c|c|c|c|c|c|}
\hline \multirow{2}{*}{$\begin{array}{l}\text { Questions } \\
\text { Choices (* indicates the right one) }\end{array}$} & \multirow[b]{2}{*}{$a^{*}$} & \multicolumn{2}{|c|}{ Q1 } & \multirow[b]{2}{*}{$\mathrm{d}$} & \multicolumn{3}{|c|}{$\mathrm{Q} 2$} & \multirow[b]{2}{*}{$\mathrm{d}$} \\
\hline & & $\mathrm{b}$ & $\mathrm{c}$ & & $a^{*}$ & $\mathrm{~b}$ & $\mathrm{c}$ & \\
\hline Students' answers $(N=94)$ & $89 \%$ & $0 \%$ & $0 \%$ & $11 \%$ & $89 \%$ & $0 \%$ & $0 \%$ & $11 \%$ \\
\hline $\begin{array}{l}\text { Correct justifications for the } \\
\text { right choice }(N=94)\end{array}$ & $65 \%$ & & & & $24 \%$ & & & \\
\hline Questions & & $\mathrm{Q}$ & & & & Q4 & & \\
\hline Choices ( ${ }^{*}$ indicates the right one) & $\mathrm{a}$ & $b^{*}$ & $\mathrm{c}$ & $\mathrm{d}$ & $a^{*}$ & $\mathrm{~b}$ & $\mathrm{c}$ & $\mathrm{d}$ \\
\hline Students' answers $(N=94)$ & $23 \%$ & $71 \%$ & $0 \%$ & $5 \%$ & $61 \%$ & $24 \%$ & $5 \%$ & $10 \%$ \\
\hline $\begin{array}{l}\text { Correct justifications for the } \\
\text { right choice }(N=94)\end{array}$ & & $54 \%$ & & & $33 \%$ & & & \\
\hline
\end{tabular}

Table B2. Distribution of choices and justification to Q5

\begin{tabular}{|c|c|c|c|}
\hline Choices & $N=94$ & Justifications & \\
\hline \multirow[t]{4}{*}{$\begin{array}{l}\text { (a) Yes, he perceives the flashes } \\
\text { at the same time }\end{array}$} & $49 \%$ & $\begin{array}{l}\mathrm{C} \text { and } \mathrm{E} \text { at the point of spacetime } \\
\text { where photons cross }\end{array}$ & $13 \%$ \\
\hline & & $\begin{array}{l}\text { Same place or same place } \\
\text { and same time }\end{array}$ & $15 \%$ \\
\hline & & Other & $9 \%$ \\
\hline & & None & $13 \%$ \\
\hline \multirow[t]{3}{*}{ (b) No, he perceives Alice's flash first } & $18 \%$ & Resorting to relativistic relations & $9 \%$ \\
\hline & & E comes from $\mathrm{A}$ & $6 \%$ \\
\hline & & None & $3 \%$ \\
\hline \multirow[t]{3}{*}{ (c) No, he perceives Bernard's flash first } & $18 \%$ & E goes towards B & $9 \%$ \\
\hline & & Other & $4 \%$ \\
\hline & & None & $4 \%$ \\
\hline (d) I do not know & $15 \%$ & & \\
\hline
\end{tabular}


Table B3. Distribution of choices and justification to Q7.

\begin{tabular}{|c|c|c|c|}
\hline Choices & $N=94$ & Justifications & \\
\hline \multirow[t]{3}{*}{$\begin{array}{l}\text { (a) Yes, she perceives the } \\
\text { flashes at the same time }\end{array}$} & $20 \%$ & $\begin{array}{l}\text { Same place or reference to correct justification to } \\
\text { Q3 }\end{array}$ & $9 \%$ \\
\hline & & Other & $4 \%$ \\
\hline & & None & $7 \%$ \\
\hline \multirow[t]{3}{*}{$\begin{array}{l}\text { (b) No, she perceives Alice's } \\
\text { flash first }\end{array}$} & $40 \%$ & $\begin{array}{l}\text { Same place or same place and time or reference to } \\
\text { correct justification to Q3 }\end{array}$ & $14 \%$ \\
\hline & & Other & $16 \%$ \\
\hline & & None & $10 \%$ \\
\hline $\begin{array}{l}\text { (c) No, she perceives } \\
\text { Bernard's flash first }\end{array}$ & $7 \%$ & & \\
\hline $\begin{array}{l}\text { (d) I do not know or e) no } \\
\text { answer }\end{array}$ & $33 \%$ & & \\
\hline
\end{tabular}

Table B4. Distribution of answers to Q6 and Q8.

\begin{tabular}{|c|c|c|c|}
\hline Choices of answers & $N=94$ & Justifications & \\
\hline \multirow[t]{3}{*}{$\begin{array}{l}\text { Students who tick box (b) or } \\
\text { (c) to both Q6 and Q8 }\end{array}$} & $23 \%$ & $\begin{array}{l}\text { Correct justification } \\
\text { box (c) ticked. }\end{array}$ & $3 \%$ \\
\hline & & $\begin{array}{l}\text { Justification where the } \\
\text { order of perception governs } \\
\text { the order of emission }\end{array}$ & $6 \%$ \\
\hline & & Without justification & $14 \%$ \\
\hline \multirow{4}{*}{$\begin{array}{l}\text { Students who tick } \\
\text { (a) to both Q6 and Q8 }\end{array}$} & $20 \%$ & Justification using the classical & $8 \%$ \\
\hline & & kinematic framework & \\
\hline & & Without justification & $8 \%$ \\
\hline & & Others & $4 \%$ \\
\hline Students who do not answer Q6 & $23 \%$ & & \\
\hline Students who do not answer Q8 & $41 \%$ & & \\
\hline
\end{tabular}

\section{References}

Artigue M 1994 Didactical engineering as a framework for the conception of teaching products ed Biehler et al Didactics of Mathematics as a Scientific Discipline (Dordrecht: Kluwer) pp 27-39

Dimitriadi K, Halkia K and Skordoulis C 2009 An attempt to teach the theory of special relativity to students of upper secondary education ESERA 2009 Conf. (Istanbul, Turkey, 31 August-4 September 2009)

Einstein A and Infeld L 1938 Evolution of Physics. From Early Concepts to Relativity and Quanta (New York: Touchstone)

Hewson P W 1982 A case study of conceptual change in special relativity: the influence of prior knowledge in learning Int. J. Sci. Educ. 4 61-78

Saltiel E and Malgrange J L 1980 Spontaneous ways of reasoning in elementary kinematics Eur. J. Phys. 173-80

Savage C M, Searle A and McCalman L 2007 Real time relativity: exploration learning of special relativity Am. J. Phys. 75 791-8 
Scherr R 2007 Modeling student thinking: an example from special relativity Am. J. Phys. 75 272-80

Scherr R, Schaffer P and Vokos S 2001 Student understanding of time in special relativity: simultaneity and references frames Am. J. Phys. 69 24-35

Strauss A and Corbin J 1990 Basics of Qualitative Research: Grounded Theory Procedures and Techniques (Newbury Park, CA: Sage)

Villani A and Pacca J L A 1987 Students' spontaneous ideas about the speed of light Int. J. Sci. Educ. 9 55-66 